\title{
Spontaneous Complete Necrosis of Hepatocellular Carcinoma: Possible Immune System Hypothesis
}

\author{
Radwan Kassir ${ }^{\mathrm{a}, \mathrm{d}}$, Gabriele Barabino ${ }^{\mathrm{a}}$, Serban Bageacu ${ }^{\mathrm{a}}$, Michel Peoc'h ${ }^{\mathrm{b}}$, \\ Michele Jouffre-Cottierc, Jack Porcheron ${ }^{\text {a }}$
}

\begin{abstract}
Spontaneous regression of hepatocellular carcinoma (HCC) is an extraordinarily unusual phenomenon. The mechanisms underlying this intriguing phenomenon remain unknown. We report a 72-yearold man with a liver mass. MR pictures demonstrate arterial hypervascularity with venous washout. We performed a fine-needle aspiration cytology of this nodule and it allowed us to find an HCC well differentiated. Postoperative histopathologic findings demonstrated a spontaneous complete necrosis of HCC. The necrotic tissue was filled with large debris from a neoplastic tissue, regardless of no viable tumor cells were seen among them. The patient is alive and well for 2 years after surgery. The underlying mechanism of this phenomenon is not clear. The histologic findings of our patient are including the tumor necrosis, the severe inflammatory infiltration with lymphocytes and the thick fibrous capsule. We speculate that some immune mechanism may be involved in regression of HCC.
\end{abstract}

Keywords: Spontaneous regression; Hepatocellular carcinoma; MRI scan; Surgery; Immune mechanism

\section{Introduction}

Hepatocellular carcinoma (HCC) usually exhibits a poor

Manuscript accepted for publication December 3, 2013

${ }^{a}$ Department of Digestive Surgery, CHU NORD, Jean Monnet University, Saint Etienne, France

${ }^{b}$ Department of Pathology, CHU NORD, Jean Monnet University, Saint Etienne, France

${ }^{c}$ Department of Histology and Embryology, CHU NORD, Jean Monnet University, Saint Etienne, France

${ }^{\mathrm{d}}$ Corresponding author: Radwan Kassir, Department of Digestive Surgery, CHU Hospital, Jean Monnet University, Avenue Albert Raimond, 42270 Saint Etienne, France.

Email: radwankassir42@hotmail.Fr

doi: http://dx.doi.org/10.14740/jcs204w prognosis. Spontaneous regression of HCC is an extraordinarily unusual phenomenon. The underlying mechanism of this phenomenon is not clear but several factors have been proposed including deficit of vitamin $\mathrm{K}$, blood transfusion, alcohol, use of herbal medications, anti-estrogen therapy and an upper gastrointestinal hemorrhage.

\section{Case Report}

A 72-year-old man consulted for vague abdominal pains. We did not note any particular antecedent. The patient does not have any risk factors for development of HCC. We also did not note any medicamentous catching, any tobacco addiction, nor alcohol consumption. The clinical and biologic examinations were perfectly normal. The abdominal ultrasonography and the hepatic MRI have discerned a nodular lesion measuring $14 \mathrm{~mm}$ in the VII segment (Fig. 1, 2). The findings of MRI are compatible to those of typical HCC. HCC tumors derive blood flow predominantly from the hepatic artery and tend to enhance during the arterial phase or 2 - $40 \mathrm{sec}$ after contrast infusion.

We performed a fine-needle aspiration cytology of this

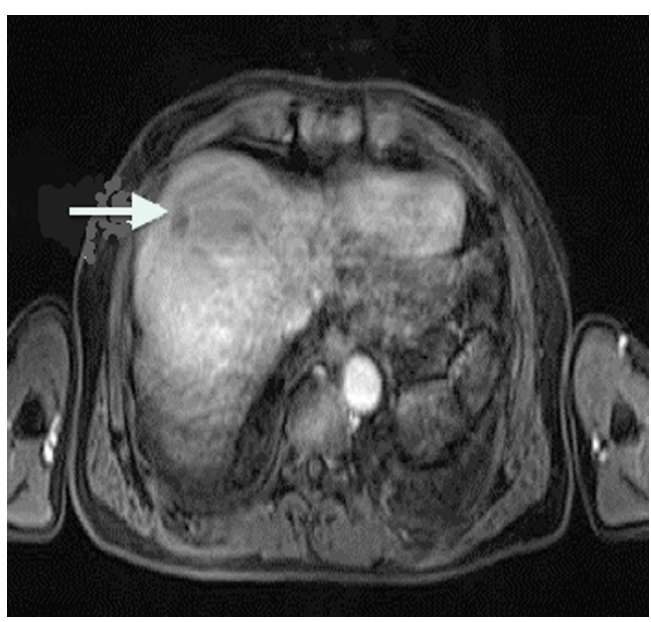

Figure 1. Hepatic MRI. Nodular lesion in the VII segment (T1 VIBE sequence). 


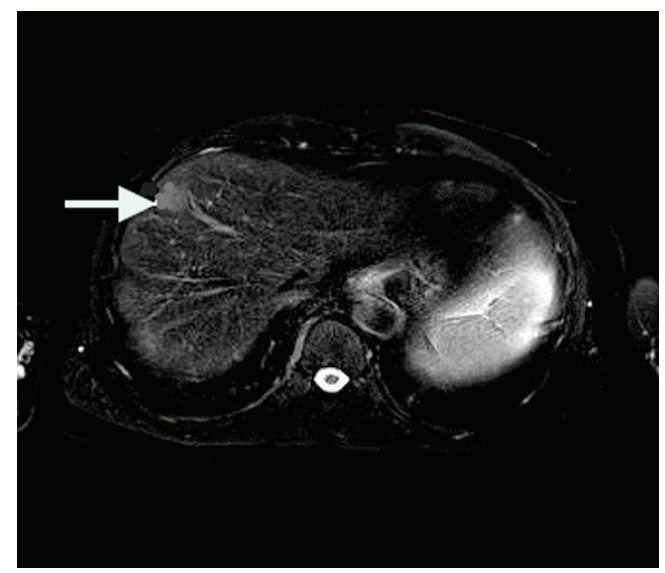

Figure 2. Hepatic MRI. Nodular lesion in the VII segment (T2 sequence).

nodule and it allowed us to find an HCC well differentiated (Fig. 3). The case was discussed by the multidisciplinary oncologic team who decided that tumorectomy by coelioscopy should be performed. Two month later, the tumorectomy has been made with ultracision ACE, in alternation with the bipolar grip and the operational continuations were simple. The anatomopathologic results (HE staining) have revealed a complete tumor necrosis outside and the hepatic architecture is normal with an arc shape lymphocytic band, and margin was $1 \mathrm{~cm}$ along the specimen (Fig. 4). Microscopically, the resected mass did not contain any malignant cells.

Therefore, the histologic examination has concluded that it was a completely necrotic nodule developed on a healthy liver and the ablation has been complete. We have chosen to do a simple surveillance.

Six months later, the abdominal CT scan has not found any mass on the ablation area. The patient is alive three years after the surgery without evidence of recurrence.

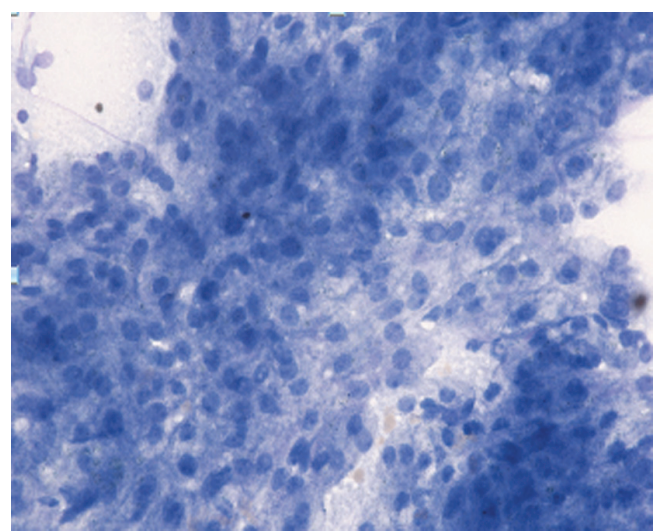

Figure 3. Fine-needle aspiration cytology (May-GrunwaldGiemsa stain, $\times 400$ ). Cluster of tumor cells resembling hepatocytes with spherical nuclei and conspicuous nucleoli.

\section{Discussion}

Spontaneous regression of $\mathrm{HCC}$ is an extraordinarily unusual phenomenon. Spontaneous regression has been defined by Everson and Cole as the partial or complete involution of a malignant tumor without application of a specific therapy [1]. The incidence of spontaneous regression has been estimated to be one in every 140,000 cases of cancer [2]. In 1956, Lee et al first reported spontaneous regression of a massive hepatica in a 5.5-month-old male infant [3].

We have reported a very rare case of spontaneous regression of HCC with complete necrosis. Complete regression of HCC was confirmed by examination of the surgically resected specimens. The mechanisms underlying this intriguing phenomenon remain unknown [4]. Possible mechanisms discussed in the literature originate primarily from the analysis of individual case histories because of the rarity of this phenomenon. The underlying mechanism of
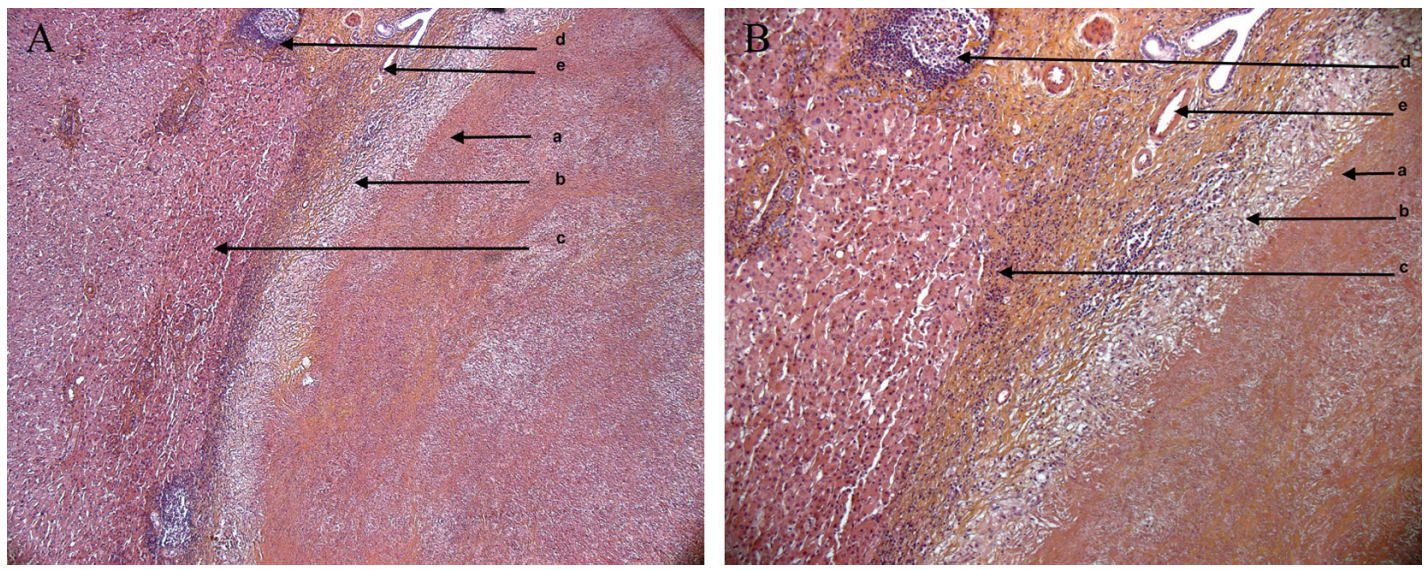

Figure 4. $(A, B)$ The anatomopathologic results (HE staining). Complete necrose of the tumor. $a=$ necrose of the tumor. $b=$ macrophages band and inflammatory granuloma. $c=$ normal hepatocytes. $d=$ biliary duct. $e=$ vessel. 
this phenomenon is not clear but several factors have been proposed including deficit of vitamin $\mathrm{K}$, blood transfusion, alcohol, use of herbal medications, anti-estrogen therapy [5] and an upper gastrointestinal hemorrhage [6]. Kojima et al have reported the case of a patient with HCC with multiple lung metastases in whom malignancy spontaneously regressed after taking Pheliinus linteus Mycelium [7]. Because of the hypervascular nature of HCC, another important factor might be an insufficient blood supply to the tumor. The ischemia of $\mathrm{HCC}$ due to disruption of the feeding artery might have induced tumor regression in the case reported by Okano et al [8].

In our case, the reasons why resected specimen showed complete tumor necrosis remain unknown. The immune system is the possible reason why resected specimen showed complete tumor necrosis. This patient had not received any medication, and there were no evident events or changes in this habitis during this period. The most frequent mechanism explaining the spontaneous disappearance of HCC is immunologic and we think that some of the characteristics observed in our case may well fit this model. Peritumoral lymphocyte infiltration with pseudocapsular lining has been described in a case of spontaneously regressing HCC [9]. This result has also been described in our case. Spontaneous regression seemed to occur during interval between percutaneous fine-needle aspiration and tumor resection existed. The histologic findings of our patient are including the tumor necrosis, the severe inflammatory infiltration with lymphocytes and the thick fibrous capsule. Therefore, some immune mechanism may be involved in regression of HCC. To our knowledge, there has been only one report of two cases of recurrence of HCCs after spontaneous regression.

\section{Conclusion}

A rare case of spontaneous regression of $\mathrm{HCC}$ with complete necrosis is presented. The changes in the vascular supply as well as in the immune system demonstrate good anticancer effects. We can speculate that complete spontaneous necrosis of HCC can promote the spontaneous regression of HCC.

\section{Conflict of Interest}

The authors declare no conflict of interest.

\section{References}

1. Beasley RP. Hepatitis B virus as the etiologic agent in hepatocellular carcinoma epidemiologic considerations. Hepatology. 1982;73:383-394.

2. Meza-Junco J, Montano-Loza AJ, Martinez-Benitez B, Cabrera-Aleksandrova T. Spontaneous partial regression of hepatocellular carcinoma in a cirrhotic patient. Ann Hepatol. 2007;6(1):66-69.

3. Lee CM, Jr., Newstedt JR, Siddall HS. Large abdominal tumors of childhood; other than Wilms' tumor or neuroblastoma. Ann Surg. 1956;143(6):803-813; discussion, 814-805

4. Ikeda M, Okada S, Ueno H, Okusaka T, Kuriyama H. Spontaneous regression of hepatocellular carcinoma with multiple lung metastases: a case report. Jpn J Clin Oncol. 2001;31(9):454-458.

5. O'Beirne JP, Harrison PM. The role of the immune system in the control of hepatocellular carcinoma. Eur J Gastroenterol Hepatol. 2004;16(12):1257-1260.

6. Bastawrous S, Kogut MJ, Bhargava P. Spontaneous regression of hepatocellular carcinoma in a cirrhotic patient: possible vascular hypothesis. Singapore Med J. 2012;53(10):e218-221.

7. Kojima H, Tanigawa N, Kariya S, Komemushi A, Shomura Y, Sawada S, Arai E, et al. A case of spontaneous regression of hepatocellular carcinoma with multiple lung metastases. Radiat Med. 2006;24(2):139-142.

8. Okano A, Ohana M, Kusumi F, Nabeshima M. Spontaneous Regression of Hepatocellular Carcinoma due to Disruption of the Feeding Artery. Case Rep Oncol. 2013;6(1):180-185.

9. Markovic S, Ferlan-Marolt V, Hlebanja Z. Spontaneous regression of hepatocellular carcinoma. Am J Gastroenterol. 1996;91(2):392-393. 